\section{Expresión de microARNs circulantes como opción para la detección de cáncer de próstata}

Rodríguez-Báez A, ${ }^{1}$ Comoto-Santacruz DA,${ }^{1}$ Huerta-Núñez LFE, ${ }^{1}$ CamposSaucedo JG, ${ }^{2}$ Estrada-Carrasco $\mathrm{CE}^{2}$

\section{Resumen}

ANTECEDENTES: los microARNs (miARNs) son moléculas alteradas del tejido tumoral prostático, incluso en casos de resistencia farmacológica. Los miARNs son importantes reguladores de la expresión génica que participan en varios procesos patológicos. Estas moléculas, detectables en muestras sanguíneas, funcionan como biomarcadores de diagnóstico, pronóstico y respuesta al tratamiento del cáncer de próstata.

OBJETIVO: determinar el perfil de expresión de los miARNs mediante técnicas moleculares en pacientes con cáncer de próstata.

MATERIALES Y MÉTODOS: estudio de casos y controles, efectuado en muestras de sangre de pacientes con diagnóstico de cáncer de próstata. Extracción del ARN total del suero, síntesis de cADN, qRTPCR y análisis de la expresión de los miARNs.

RESULTADOS: se obtuvieron 20 muestras sanguíneas para análisis molecular de miARNs. El análisis de la expresión de 62 miARNs reveló que 9 tuvieron expresión alterada, de éstos 5 están reportados en la bibliografía como marcadores de cáncer de próstata resistente a la castración.

CONCLUSIÓN: la identificación de los miARNs mediante métodos no invasivos es una opción para detectar y diagnosticar cáncer de próstata y estimar el pronóstico de los pacientes con riesgo de resistencia al tratamiento farmacológico.

PALABRAS CLAVE: miARN, resistencia farmacológica, expresión génica.

Rev Mex Urol. 2017 may;77(3):199-206.

\section{Circulating miRNA expression as a clinical option for the detection of prostate cancer}

Rodríguez-Báez A, ${ }^{1}$ Comoto-Santacruz DA, ${ }^{1}$ Huerta-Núñez LFE, ${ }^{1}$ CamposSaucedo JG, ${ }^{2}$ Estrada-Carrasco $\mathrm{CE}^{2}$

\section{Abstract}

BACKGROUND: MicroRNA molecules are altered in cancerous prostate tissue. This is also true in the drug-resistant cases. MicroRNAs

\author{
${ }^{1}$ Laboratorio de Biología Molecular, Escuela Militar \\ de Graduados de Sanidad, Universidad del Ejército \\ y Fuerza Aérea. \\ ${ }^{2}$ Servicio de Urología, Hospital Central Militar. \\ SEDENA, Ciudad de México
}

Recibido: diciembre 2016

Aceptado: mayo 2017

Correspondencia

Álvaro Rodríguez Báez

alvaro_7077@hotmail.com

Este artículo debe citarse como

Rodríguez-Báez A, Comoto-Santacruz DA, HuertaNúñez LFE, Campos-Saucedo JG, Estrada-Carrasco CE. Expresión de miARNs circulantes como herramienta clínica en cáncer de próstata. Rev Mex Urol. 2017 mayo;77(3):199-206. 
are important regulators of gene expression and they are involved in various pathologic processes. These molecules have been reported as diagnostic, prognostic, and treatment response biomarkers in prostate cancer.

OBJECTIVE: To determine the microRNA expression profile through molecular biology techniques in patients with prostate cancer.

MATERIALS AND METHODS: A case-control study was conducted that analyzed blood samples from patients diagnosed with prostate cancer for serum total RNA extraction, cDNA synthesis, qRT-PCR, and microRNA expression analysis.

RESULTS: Twenty blood samples were collected for molecular miRNA study. Sixty-two microRNAs were analyzed and 9 of them were revealed to have altered expression. Five of those 9 are reported in the international literature as castration-resistant prostate cancer markers.

DISCUSSION AND CONCLUSIONS: There is clear evidence of the relation between miRNAs and their function in pathologic processes. For example, the miR-375 gene is related to proliferation and epithelial-mesenchymal transition; miR-143 is related to migration and invasion; and miR-31 is related to the androgen receptor. This could improve the biomarkers already established in prostate cancer. The identification of miRNAs through noninvasive methods is a useful tool for the detection and diagnosis of prostate cancer, as well as for the prognosis of patients at risk for developing drug resistance in relation to their treatment.

KEY WORDS: MiRNA; Drug resistance; Gene expression

\footnotetext{
${ }^{1}$ Laboratorio de Biología Molecular, Escuela Militar de Graduados de Sanidad, Universidad del Ejército y Fuerza Aérea.

${ }^{2}$ Servicio de Urología, Hospital Central Militar.

SEDENA, Ciudad de México
}

Correspondence

Álvaro Rodríguez Báez

alvaro_7077@hotmail.com

\section{ANTECEDENTES}

El cáncer de próstata es el tumor maligno más frecuente en hombres mayores de 50 años de edad. Esta neoplasia representa la primera causa de muerte en varones de México.

A partir del decenio de 1980, con la introducción del antígeno prostático específico, se ha logrado establecer el diagnóstico en etapas más tempranas y con ello implementar un tratamiento potencialmente curativo. ${ }^{1}$

El porcentaje de hombres adultos, de 60 años de edad o mayores, a quienes se realizó la prueba de detección de cáncer de próstata por medio del antígeno prostático se mantuvo constante entre 2006 y 2012 (10.4 vs 9.5\%, respectivamente). Incluso se estimó que $2.5 \%$ de los hombres iguales o mayores de 20 años de edad acudieron en los 12 meses previos a la encuesta ENSANUT 2012 a realizarse la prueba de tacto rectal. ${ }^{2}$

Un reto importante en la práctica clínica es la incapacidad de las pruebas de diagnóstico actuales, incluido el antígeno prostático específico y la clasificación histopatológica, para distinguir entre tumores agresivos e indolentes. ${ }^{3} \mathrm{El}$ antígeno prostático específico puede detectarse en las secreciones prostáticas normales y sus concentraciones suelen elevarse en pacientes con cáncer de próstata. ${ }^{4,5}$ Durante más de 20 años las 
concentraciones séricas de antígeno prostático se han utilizado como un biomarcador del cáncer de próstata, lo que ha revolucionado el tratamiento clínico de la enfermedad. ${ }^{6}$ Sin embargo, tiene limitaciones inherentes, como la falta de especificidad, que resulta en sobrediagnóstico y sobretratamiento del cáncer de próstata. ${ }^{7}$

El National Cancer Institute ( $\mathrm{NCl}$ ) define "biomarcador" a una molécula biológica detectada en la sangre, fluidos corporales o tejidos, que puede determinarse y evaluarse objetivamente como resultado de un proceso biológico normal-anormal o alguna condición patógena-enfermedad.

Un biomarcador puede utilizarse para fines de selección, diagnóstico, pronóstico y evaluación de la enfermedad, además de la predicciónseguimiento de la respuesta al tratamiento para diversas intervenciones terapéuticas. ${ }^{7-10}$

Entre los biomarcadores de cáncer de próstata alternativos están los microARN (miARNs): ácidos ribonucleicos de aproximadamente 22 nucleótidos de longitud, no codificantes, importantes reguladores de la expresión génica en células eucariotas. ${ }^{11}$ (Figura 1).

Cada microARN tiene la capacidad de interactuar por diferentes rutas celulares; en consecuencia, los cambios en la expresión de un pequeño número de miARNs pueden reflejar la irregularidad de diversos procesos celulares, según la complejidad de la neoplasia. Además, los miARNs son relativamente resistentes a la degradación por la RNasa, debido a su longitud de secuencia corta, que aumenta su tiempo de vida en muestras de suero y tejidos. ${ }^{12,13}$

Las evidencias más recientes sugieren que los miARNs se encuentran en pacientes con cáncer de próstata resistentes al tratamiento farmacológico, mediante la regulación de la vía de señalización de andrógenos, apoptosis, trasportadores de fármacos, transición epiteliomesenquimal (EMT) y células madre de la neoplasia (CSC). ${ }^{14} \mathrm{El}$ receptor de andrógenos es importante en la evolución a cáncer de próstata resistente a la castración (CPRC). ${ }^{15,16}$

Los primeros estudios relacionados con la alteración de miARNs en pacientes con cáncer de próstata se reportaron entre 2007 y 2008, con el propósito de identificar los perfiles de expresión de genes para establecer el diagnóstico, pronóstico y factores predictivos. ${ }^{17,18}$

En pacientes con tumores de próstata primarios se ha informado la desregulación generalizada de miARNs comparados con tejidos sanos. La alteración de los perfiles de expresión de miARN se ha relacionado con la evolución del cáncer de próstata, agresividad, metástasis y recurrencia. ${ }^{19-21}$

Los miARNs pueden ser biomarcadores importantes en la detección del cáncer de próstata en etapas tempranas. ${ }^{22}$

El objetivo de este estudio es determinar, mediante técnicas de biología molecular, el perfil de expresión de miARNs en muestras de suero de pacientes con cáncer de próstata.

\section{MATERIALES Y MÉTODOS}

Estudio observacional, retrospectivo, transversal y comparativo, de casos y controles, en el que se evaluaron muestras sanguíneas de pacientes con diagnóstico de cáncer de próstata (grupo de casos) versus pacientes sanos (grupo control). Criterios de inclusión: sujetos con diagnóstico de cáncer de próstata primario, con indicación para prostatectomía radical, mayores de 40 años de edad, mexicanos. Criterios de exclusión: pacientes con diagnóstico diferente de cáncer de próstata. El estudio se llevó a cabo en el Hospital Central Militar, 


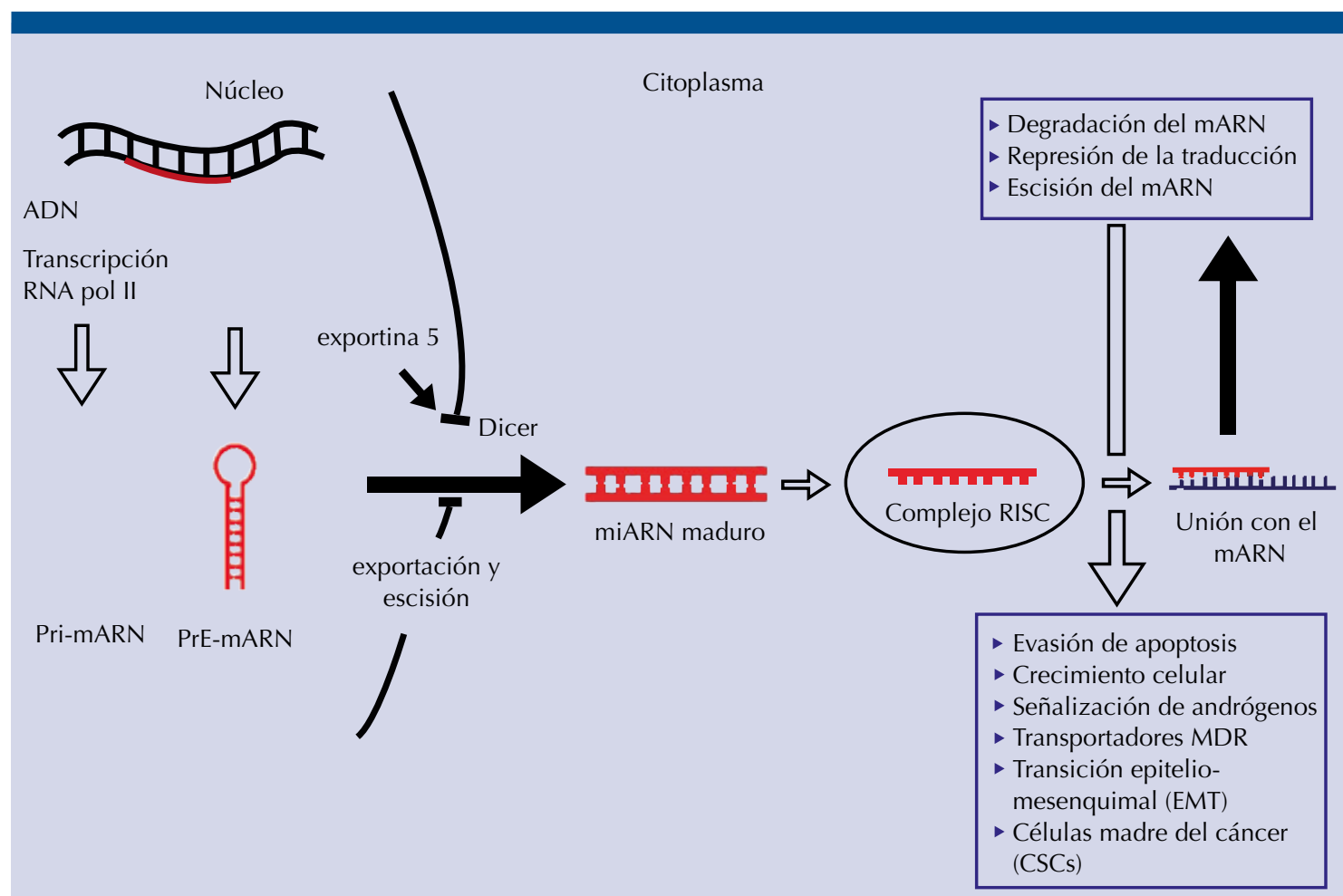

Figura 1. Expresión génica de miARNs en células eucariotas.

entre octubre de 2014 y mayo de 2016, con la aprobación del Comité de Investigación de la institución y el consentimiento informado de cada paciente.

\section{Procesamiento de las muestras}

- De cada paciente se obtuvieron $4 \mathrm{~mL}$ de sangre. Cada muestra se centrifugó y del suero se aislaron los componentes sanguíneos, para finalmente obtener $1 \mathrm{~mL}$ de éste. Las muestras se almacenaron a $-80^{\circ} \mathrm{C}$ hasta su utilización. Se colectaron $200 \mu \mathrm{L}$ de suero para obtener el ARN total mediante el método tradicional con Trizol ${ }^{\circledR}$ (Thermo Fisher Scientific). Posteriormente, el ARN extraído de cada muestra se cuantificó por espectrofotometría (Thermo scientific nanodrop 1000).

- Para la síntesis de ADN complementario (cADN) se utilizó el dispositivo miScript II $R T$ kit $^{\circledR}$ (Qiagen, Hilden, Germany), con el termociclador Veriti Thermal Cycler (Applied Biosystems). Una vez obtenido el cADN se realizaron diluciones para igualar las concentraciones en cada muestra biológica, formar grupos de cADN de cada cinco muestras y obtener como resultado final cuatro grupos de muestras de los casos (pacientes con cáncer de próstata) y tres grupos de muestras de los controles (pacientes sanos). Se realizó la amplificación (replicación) del material genético, en este caso de los 62 miARNs que actualmente 
Rodríguez-Báez A y col. Expresión de miARNs

Cuadro 1. Expresión de miARNs reportados en la bibliografía

\begin{tabular}{|c|c|c|c|c|}
\hline \multirow[t]{7}{*}{ Relación } & Nivel de expresión & \multicolumn{3}{|c|}{ miRNA } \\
\hline & & miR-135b-5p & miR-361-5p & miR-32-5p \\
\hline & & miR-15a-5p & miR-455-5p & miR-148a-3p \\
\hline & Sobreexpresados & miR-184 & miR-616-3p & miR-148a-5p \\
\hline & & TmiR-21-5p & miR-222-3p & miR-378b \\
\hline & & miR-221-3p & miR-222-5p & miR-378c \\
\hline & & miR-30c-5p & miR-32-3p & miR-378a-3p \\
\hline \multirow[t]{11}{*}{$\begin{array}{l}\text { Cáncer de próstata resistente a } \\
\text { la castración }\end{array}$} & & & & miR-378a-5p \\
\hline & & miR-100-5p & $\operatorname{miR}-27 b-3 p$ & miR-34a-3p \\
\hline & & miR-125b-5p & miR-29b-3p & miR-34c-3p \\
\hline & & miR-128-3p & miR-331-3p & miR-34c-5p \\
\hline & Subexpresados & miR-146a-5p & miR-365a-3p & miR-31-3p \\
\hline & & miR-146b-5p & miR-99a-5p & miR-31-5p \\
\hline & & miR-19b-3p & -let-7c-5p & miR-185-3p \\
\hline & & miR-203a & miR-124-5p & miR-185-5p \\
\hline & & miR-23b-3p & miR-124-3p & miR-205-5p \\
\hline & & & miR-34a-5p & miR-205-3p \\
\hline & Sobreexpresados & miR-9-3p & & \\
\hline \multirow[t]{3}{*}{$\begin{array}{l}\text { Cáncer de próstata agresivo vs } \\
\text { no agresivo }\end{array}$} & Subexpresados & miR-145-5p & miR-330-3p & \\
\hline & Expresados & miR-16-5p & & \\
\hline & & miR-141-3p & miR-19a-3p & \\
\hline \multirow[t]{3}{*}{$\begin{array}{l}\text { Marcadores séricos en sangre } \\
\text { y plasma }\end{array}$} & Sobreexpresados & miR-200b-3p & miR-19a-5p & \\
\hline & & miR-375 & & \\
\hline & & miR-143-5p & miR-34b-5p & miR-200 \\
\hline \multirow[t]{2}{*}{$\begin{array}{l}\text { Transición epitelio-mesen- } \\
\text { quimal }\end{array}$} & Expresión alterada & miR-143-3p & miR-200c-5p & miR-203a \\
\hline & & miR-34b-3p & miR-200c-3p & \\
\hline
\end{tabular}

están reportados en la bibliografía internacional relacionados con el cáncer de próstata primario, resistencia farmacológica y biomarcadores de interés (Cuadro 1), de cada grupo de muestras, mediante qRT-PCR en el termociclador Rotor-Gene Q (Qiagen, Hilden, Germany), con la finalidad de evaluar su nivel o grado de expresión.
- La cuantificación de la expresión de los miARNs identificados se determinó con la fórmula aritmética $2^{-\Delta \Delta C T}$, mediante la plataforma en línea: http://pcrdataanalysis. sabiosciences.com/mirna (Qiagen), con valor estadísticamente significativo de p >0.05 e IC95\%. 


\section{RESULTADOS}

Durante el periodo de estudio se registraron 34 pacientes: 20 asignados al grupo de casos (diagnóstico de cáncer de próstata) y 14 al grupo control (sanos).

El análisis de la expresión de los 62 miARNs, por medio de la plataforma pcrdataanalysis.sabiosciences.com/mirna, reportó 9 muestras con expresión alterada, de las que cinco están reportadas como marcadores de cáncer de próstata resistente a la castración (CPRC): miR-32-5p, miR455-4p, miR-184, miR-31-5p y miR-200b-3p, 3 relacionados con apoptosis: miR-19b-3p, miR$34 a-5 p$, miR-32-5p y 3 relacionados con EMT: miR-143-5p, miR-200b-3p y miR-375 (Cuadro 2).

\section{DISCUSIÓN}

En este estudio se logró aislar, detectar y determinar la expresión diferencial de miARNs en muestras sanguíneas de pacientes con cáncer de próstata versus sujetos sanos.

Los miARNs identificados extracelularmente en diversos fluidos corporales, incluida la sangre (suero), se asocian con proteínas y lipoproteínas, empaquetados dentro de las estructuras celulares (exosomas, microvesiculas o cuerpos apoptósicos). ${ }^{23}$

Algunos estudios reportan distintos perfiles de expresión de miARNs alterados en el tejido tumoral prostático versus tejido sano. 22,24

Brase y su grupo, en su estudio con muestras de suero de pacientes con cáncer de próstata metastásico $v s$ localizado $(n=14)$, encontraron 5 miARNs sobrexpresados (miR-375, miR-9, miR-200b, miR-141 y miR-516a-3p), que posteriormente se validaron, además de identificar miR-375 y miR-141 como marcadores de alto riesgo de cáncer de próstata. ${ }^{25}$

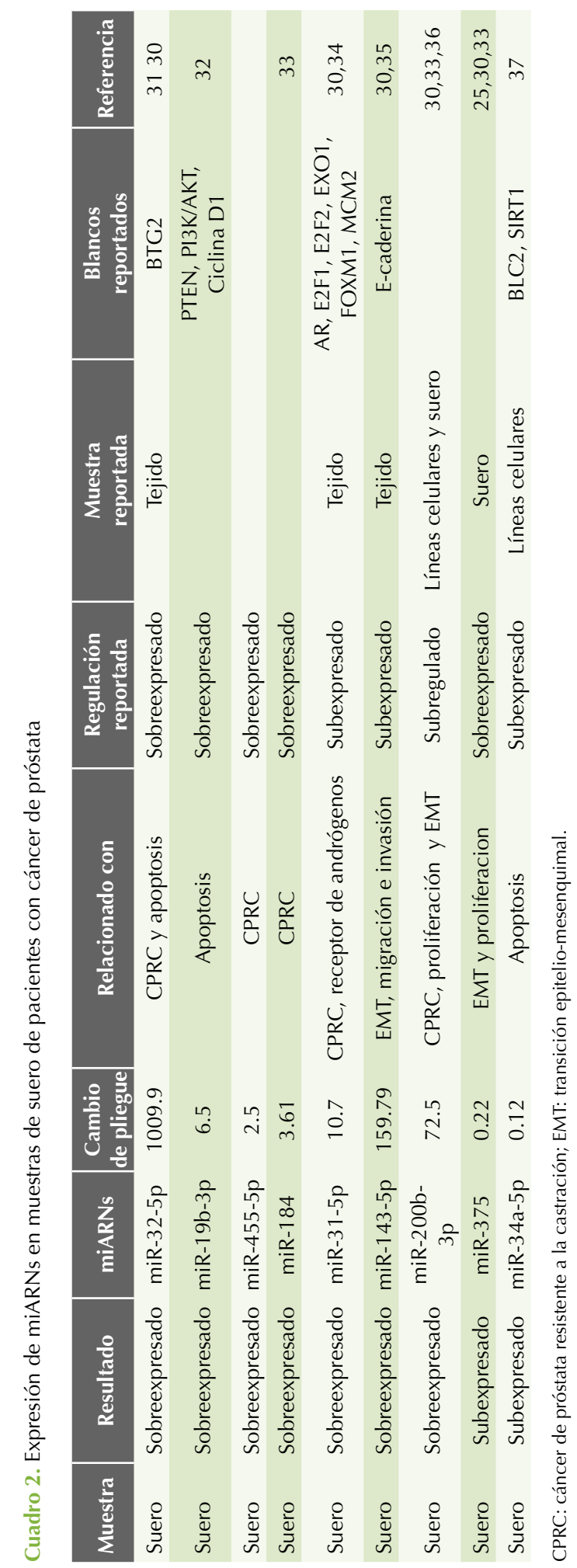


En el estudio aquí comunicado se identificaron 9 miARNs de los que siete se encontraron sobrexpresados (miR-32-5p, miR-19b-3p, miR-455-5p, miR-184, miR-31-5p, miR-143-5p, miR-200b$3 p)$ y 2 subexpresados (miR-375, miR-34a), teniendo en común con otros estudios el gen miR-375.

Un ensayo efectuado por microarreglos y reacción en cadena de la polimerasa-transcriptasa reversa, cuyos perfiles de miARNs se analizaron en el suero de un modelo de ratón de cáncer de próstata, identificaron como biomarcadores de la enfermedad: miR-141, miR-298, miR-346 y miR-375. ${ }^{26}$ En el estudio de Lodes y sus colaboradores, también confirmaron la expresión de miR-141 y miR-375 en el suero y plasma de pacientes con cáncer de próstata, respectivamente. $^{27}$

De acuerdo con otros estudios, el análisis de expresión alterada de miARNs en pacientes con cáncer de próstata reveló que los genes miR-141, miR-200b y miR-375 participan en el control de la expresión del receptor de andrógenos, la plasticidad de la membrana celular ${ }^{28}$ y la reparación del $A D N$, respectivamente. ${ }^{29}$ Estos datos coinciden con los genes miR-200b y miR-375 detectados en nuestro estudio.

Una revisión demostró que los genes: miR-375 y miR-200b se relacionan con la proliferación celular, miR-375 se asocia con la transición epitelio-mesenquimal, miR-143 con la migración e invasión, miR-31 con el receptor de andrógenos y miR-32 con la apoptosis. ${ }^{30}$

Es importante continuar con la investigación e identificación de miARNs como biomarcadores, con la finalidad de incrementar la sensibilidad y especificidad de las pruebas diagnósticas actuales para poder disponer de una técnica complementaria para diagnosticar pacientes con cáncer de próstata.

\section{CONCLUSIONES}

La identificación de miARNs, mediante métodos no invasivos, es una técnica útil para establecer, oportunamente, el diagnóstico de cáncer de próstata y, de esta manera, evaluar el pronóstico de los pacientes con riesgo de resistencia al tratamiento farmacológico.

\section{Financiamiento}

Proyecto A022 "Investigación y desarrollo militar en coordinación con universidades, instituciones de educación superior y otros centros públicos de investigación".

\section{Conflicto de intereses}

Los autores declaran no tener conflictos de interés.

\section{REFERENCIAS}

1. Jiménez-Ríos $M$, Solares-Sánchez $M$, Martínez-Cervera P, Aguilar-Ponce J, Martínez-Cedillo J, Hinojosa-Gómez J. Oncoguía: Cáncer de próstata. Cancerología 2011;6:13-18.

2. Gutiérrez JP, Rivera-Dommarco J, Shamah-Levy T, et al. Encuesta Nacional de Salud y Nutrición 2012. Resultados nacionales, Cuernavaca, México: Instituto Nacional de Salud Pública 2012:1-196.

3. Otero JR, Gómez BG, Juanatey FC, Touijer KA. Prostate cancer biomarkers: An update. Urologic oncology: Seminars and original investigations. Urol Oncol 2014:252-60.

4. Lilja H. Testing new PSA subforms to enhance the accuracy of predicting cancer risk and disease outcome in prostate cancer. Clin Chem 2008; 54:1248-9.

5. Lilja H, Ulmert D, Vickers AJ. Prostate-specific antigen and prostate cancer: prediction, detection and monitoring. Nature Rev Cancer 2008;8:268-78.

6. Cary KC, Cooperberg MR. Biomarkers in prostate cancer surveillance and screening: past, present, and future. Ther Adv Urol 2013;5:318-29.

7. Saini S. PSA and beyond: alternative prostate cancer biomarkers. Cell Oncol 2016;39:97-106.

8. Sawyers $\mathrm{CL}$. The cancer biomarker problem. Nature 2008;452:548-52.

9. Prensner JR, Rubin MA, Wei JT, Chinnaiyan AM. Beyond PSA: the next generation of prostate cancer biomarkers. Sci Translat Med 2012;4:127rv3-rv3. 
10. Ilyin SE, Belkowski SM, Plata-Salamán CR. Biomarker discovery and validation: technologies and integrative approaches. Trends Biotech 2004;22:411-6.

11. Graves P, Zeng Y. Biogenesis of mammalian microRNAs: a global view. Genomics Proteomics Bioinformatics 2012;10:239-45.

12. Chen $X, B a Y, M a L$, et al. Characterization of microRNAs in serum: a novel class of biomarkers for diagnosis of cancer and other diseases. Cell Res 2008;18:997-1006.

13. Jackson BL, Grabowska A, Ratan HL. MicroRNA in prostate cancer: functional importance and potential as circulating biomarkers. BMC Cancer 2014;14:1.

14. Li F, Mahato RI. MicroRNAs and drug resistance in prostate cancers. Mol Pharm 2014;11:2539-52.

15. Massard C, Fizazi K. Targeting continued androgen receptor signaling in prostate cancer. Clin Cancer Res 2011;17:3876-83.

16. Kim WT, Kim W-J. MicroRNAs in prostate cancer. Prostate international 2013;1:3-9.

17. Ozen M, Creighton C, Ozdemir M, Ittmann M. Widespread deregulation of microRNA expression in human prostate cancer. Oncogene 2008;27:1788-93.

18. Porkka KP, Pfeiffer MJ, Waltering KK, Vessella RL, Tammela TL, Visakorpi T. MicroRNA expression profiling in prostate cancer. Cancer Res 2007;67:6130-5.

19. Gordanpour A, Nam R, Sugar L, Seth A. MicroRNAs in prostate cancer: from biomarkers to molecularly-based therapeutics. Prostate Cancer Prostatic Dis 2012;15:314-9.

20. Hurst DR, Edmonds MD, Welch DR. Metastamir: the field of metastasis-regulatory microRNA is spreading. Cancer Res 2009;69:7495-8.

21. Saini S, Majid S, Dahiya R. Diet, microRNAs and prostate cancer. Pharm Res 2010;27:1014-26.

22. Szczyrba J, Löprich E, Wach S, et al. The microRNA profile of prostate carcinoma obtained by deep sequencing. Mol Cancer Res 2010;8:529-38.

23. Hessvik NP, Sandvig K, Llorente A. Exosomal miRNAs as biomarkers for prostate cancer. Front Genet 2014;21:36.

24. Ambs S, Prueitt RL, Yi M, et al. Genomic profiling of microRNA and messenger RNA reveals deregulated microRNA expression in prostate cancer. Cancer Res 2008;68:6162-70.
25. Brase JC, Johannes M, Schlomm T, et al. Circulating miRNAs are correlated with tumor progression in prostate cancer. Int J Cancer 2011;128:608-16.

26. Selth LA, Townley S, Gillis JL, et al. Discovery of circulating microRNAs associated with human prostate cancer using a mouse model of disease. Int J Cancer 2012;131:652-61.

27. Lodes MJ, Caraballo M, Suciu D, Munro S, Kumar A, Anderson B. Detection of cancer with serum miRNAs on an oligonucleotide microarray. PLoS One 2009;4:e6229.

28. Williams LV, Veliceasa D, Vinokour E, Volpert OV. miR-200b inhibits prostate cancer EMT, growth and metastasis. PLoS One 2013;8:e83991.

29. Costa-Pinheiro P, Montezuma D, Henrique R, Jerónimo $C$. Diagnostic and prognostic epigenetic biomarkers in cancer. Epigenomics 2015;7:1003-15.

30. Bertoli G, Cava C, Castiglioni I. MicroRNAs as biomarkers for diagnosis, prognosis and theranostics in prostate cancer. Int J Mol Sci 2016;17:421.

31. Jalava S, Urbanucci A, Latonen L, et al. Androgen-regulated miR-32 targets BTG2 and is overexpressed in castrationresistant prostate cancer. Oncogene 2012;31:4460-71.

32. Tian L, Fang $Y X, X u e ~ J L, C h e n ~ J Z$. Four microRNAs promote prostate cell proliferation with regulation of PTEN and its downstream signals in vitro. PloS one 2013;8:e75885.

33. Haldrup C, Kosaka N, Ochiya T, et al. Profiling of circulating microRNAs for prostate cancer biomarker discovery. Drug Deliv TransI Res 2014;4:19-30.

34. Lin P-C, Chiu Y-L, Banerjee S, et al. Epigenetic repression of miR-31 disrupts androgen receptor homeostasis and contributes to prostate cancer progression. Cancer Res 2013;73:1232-44.

35. Peng X, Guo W, Liu T, et al. Identification of miRs-143 and145 that is associated with bone metastasis of prostate cancer and involved in the regulation of EMT. PloS One 2011;6:e20341.

36. He M, Liu Y, Deng X, et al. Down-regulation of miR-200b-3p by low $p 73$ contributes to the androgen-independence of prostate cancer cells. Prostate 2013;73:1048-56.

37. Kojima K, Fujita Y, Nozawa Y, Deguchi T, Ito M. MiR-34a attenuates paclitaxel-resistance of hormone-refractory prostate cancer PC3 cells through direct and indirect mechanisms. Prostate 2010;70:1501-12. 